\title{
Variational study of the interacting, spinless Su-Schrieffer-Heeger model
}

\author{
M Yahyavi ${ }^{1,3}{ }^{10}$, L Saleem $^{1,3}$ and B Hetényi ${ }^{1,2}{ }^{10}$ \\ 1 Department of Physics, Bilkent University, TR-06800 Bilkent, Ankara, Turkey \\ 2 MTA-BME Exotic Quantum Phases 'Momentum' Research Group, Department of Physics, \\ Budapest University of Technology and Economics, H-1111 Budapest, Hungary \\ E-mail: m.yahyavi@bilkent.edu.tr, luqman.saleem@bilkent.edu.tr, hetenyi@fen.bilkent.edu.tr \\ and hetenyi@phy.bme.hu
}

Received 20 June 2018, revised 7 September 2018

Accepted for publication 12 September 2018

Published 11 October 2018

\begin{abstract}
We study the phase diagram and the total polarization distribution of the Su-SchriefferHeeger model with nearest neighbor interaction in one dimension at half-filling. To obtain the ground state wave-function, we extend the Baeriswyl variational wave function to account for alternating hopping parameters. The ground state energies of the variational wave functions compare well to exact diagonalization results. For the case of uniform hopping for all bonds, where it is known that an ideal conductor to insulator transition takes place at finite interaction, we also find a transition at an interaction strength somewhat lower than the known value. The ideal conductor phase is a Fermi sea. The phase diagram in the whole parameter range shows a resemblance to the phase diagram of the Kane-Mele-Hubbard model. We also calculate the gauge invariant cumulants corresponding to the polarization (Zak phase) and use these to reconstruct the distribution of the polarization. We calculate the reconstructed polarization distribution along a path in parameter space which connects two points with opposite polarization in two ways. In one case we cross the metallic phase line, in the other, we go through only insulating states. In the former case, the average polarization changes discontinuously after passing through the metallic phase line, while in the latter the distribution 'walks across' smoothly from one polarization to its opposite. This state of affairs suggests that the correlation acts to break the chiral symmetry of the Su-Schrieffer-Heeger model, in the same way as it happens when a Rice-Mele onsite potential is turned on.
\end{abstract}

Keywords: correlated electrons, topological insulators, one-dimensional systems

(Some figures may appear in colour only in the online journal)

\section{Introduction}

The study of topological systems [1,2] is an extremely active research area. Recent efforts [3] have focused on understanding the effects of electron interaction on such systems. Part of this effort concentrates on extending [4-7] the symmetry classification valid for non-interacting systems [8,9], part of it is to map the phase diagrams of existing topological models with interaction turned on $[10,11]$. One model which has received considerable attention is the Kane-Mele-Hubbard

\footnotetext{
${ }^{3}$ Authors contributed equally.
}

(KMH) model [12] both analytically [13, 14] and numerically $[15,16]$.

The starting point in topological analyses is usually the Berry phase [17-19]. The Berry phase which arises from integrating across the Brillouin zone [20] (Zak phase) corresponds to the polarization [21-23] of a crystalline system, while its modified versions give topological indices such as the Chern number [24] or time-reversal polarization [25]. The Zak phase can be viewed as the first in a series of gauge invariant cumulants [26-28], the second corresponding [29] to the variance in the center of mass of the electronic charge distribution.

Recently, higher order cumulants were studied [28, 30, 31]. It was shown [30] that the third cumulant, also known as the 
skew, corresponds to the so called shift current, the secondorder nonlinear optical response in second harmonic generation experiments (this work addresses such experiments in a Weyl semimetal). It was emphasized, that the skew gives a more intuitive picture of the system, than the sum rules valid for nonlinear response. Kobayashi et al [31] study the quantity $Z^{(q)}=\langle\Psi|\exp (\mathrm{i} 2 \pi \hat{X} / L) q| \Psi\rangle(L$ denotes the system size, $\hat{X}$ is the total position operator, and $q$ is a real number, integer in systems with periodic boundary conditions) in the metallic phase. The $q=1$ case was suggested by Resta and Sorella $[23,29]$ for the polarization and its variance. A modification to this scheme was suggested by Aligia and Ortiz [32] for lattice systems with fractional fillings. It can be shown that for general $q$ this quantity contains the same information as the gauge invariant cumulants. In [31] it is shown that the size dependence of $Z^{(q)}$ in the metallic phase is universal. From the first six cumulants the distribution of the polarization in the Rice-Mele (RM) model [33] was reconstructed [28].

In this paper we study the $1 \mathrm{D}$ spinless interacting $\mathrm{Su}-$ Schrieffer-Heeger (SSH) model [34]. This model was studied via a diagrammatic expansion [36] and the density-matrix renormalization group [35]. The spinful version of this model was studied by Manmana et al [37]. Via a Jordan-Wigner transformation this model can be transformed into an interacting spin model, which falls into a group of models known as the alternating bond spin- $-\frac{1}{2}$ Heisenberg models. Bond alternation opens a gap $[38,39]$ in spin- $\frac{1}{2}$ systems, which is analogous to the fact that the SSH model is insulating. The study of such models already has a long history [40, 41], recently $[42,43]$ there has been renewed interest due to their topological phases [42, 43]. In [42] the topological behavior gauged via a string order parameter.

We develop a variational approach by extending the Baeriswyl wave function [44, 45] (BWF) to account for the alternating hoppings of the SSH. We compare the ground state energies to exact diagonalization results for small system sizes, finding excellent agreement. The phase diagram we find is remarkably similar to that of the KMH model [3, 12]. When all hopping parameters are equal, we find a conducting phase for small interaction (in our variational treatment a Fermi sea), and a correlated insulator with charge density wave ordering for large interaction. When the hoppings alternate, the small interaction phase is the SSH ground state, but as the interaction increases, the system tends towards charge density order, which is weakened by the hopping alternation. We also construct a parent Hamiltonian for the BWF type wave function we use. Our construction allows for plotting the curves traced out by varying $k$ across the Brillouin zone in the space spanned by the components of the Hamiltonian.

We then study the behavior of the polarization distribution. In particular, we do reconstructions along two paths in the parameter space of the model, which connect topologically distinct states. Both paths connect two states with finite interaction parameter, but with opposite polarity in hopping. One path crosses the metallic phase line (the interaction parameter is constant), while the other passes through the insulating regime only (the interaction is varied). We find that in the first case, the maximum of the distribution remains constant until the metallic phase line is reached, there the distribution flattens, and after passing to the other side of the metallic phase line, the distribution has a maximum at a different polarization (a jump occurred in the Berry phase at the metallic phase line). For the second path, the maximum, as well as the other cumulants vary smoothly, the distribution 'walks across' from one polarization to the other. To make sense of these results, we relate them to the well-known topological quantum phase transition which occurs in the SSH model. In the SSH model chiral symmetry gives rise to a symmetry protected topological phase separated from a topologically trivial phase by gap closure. Again we reconstruct the polarization distribution along two different paths. Both paths pass between topologically distinct phases, but along one the symmetries are always respected (gap closure occurs), while along the other, the symmetries are relaxed. The polarization distributions evolve exactly in the same manner.

Our paper is organized as follows. In the following section we give the models we study, the form of the BWF, the construction of the parent Hamiltonian, and the gauge invariant cumulants. In section 3 our results and analyses are presented. In section 4 we conclude our work.

\section{Model and methods}

\subsection{Spinless, interacting Su-Schrieffer-Heeger model}

We study the interacting SSH model in one dimension at half filling. This model consists of a hopping parameter, which alternates between odd and even bonds, and an interaction term if two particles are on nearest neighboring sites. For the case of uniform hopping for all bonds, the model can be solved by the Bethe ansatz, and it is known that an ideal conductor (finite Drude weight) to insulator phase transition takes place.

The Hamilton operator of the model consists of two terms, the SSH term $\left(\hat{h}_{\mathrm{SSH}}\right)$ and the interaction term $\left(\hat{h}_{V}\right)$ and can be written as

$$
\begin{aligned}
\hat{H}= & \overbrace{-\frac{1}{2} \sum_{n=1}^{L}\left[t+(-1)^{n} \delta\right] c_{n}^{\dagger} c_{n+1}+\text { H.c. }}^{\hat{h}_{\text {SSH }}} \\
& +\overbrace{\frac{V}{2} \sum_{n=1}^{L} n_{n} n_{n+1}}^{\hat{h}_{V}}
\end{aligned}
$$

where $c_{n}^{\dagger}\left(c_{n}\right)$ creates (annihilates) a particle at site $n, n_{n}=c_{n}^{\dagger} c_{n}$ is the density operator at site $n$. $t$ denotes the average hopping, taken as unity in the following, $\delta$ denotes the deviation in hopping between odd and even sites. $V$ is the interaction between particles on nearest neighboring sites. This interaction would be the leading term for the full Coulomb interaction, since configurations with two particles on the same site, do not occur due to the Pauli principle. For $\delta=0$ the ideal conductor insulator transition occurs at $V=2$ in units of $t$. In the rest of the paper we use units of $t$. Figure 1 illustrates the model and the meaning of the different parameters. 


\subsection{The variational wave function}

The BWF [44-46, 48] already has a history of successfully reproducing the properties of strongly correlated models. It was originally developed for the fermionic Hubbard model, but it has been applied to the bosonic Hubbard [49] as well as quenches interacting spinless fermions [50].

In this section we describe the BWF $[44,45]$, its extensions necessary to account for the alternating hoppings of the SSH model, and write down the solution for the variational energy in closed form.

The BWF starts with the wave function at infinite interaction (a charge density wave) and acts on this wave function with a kinetic energy based projector. In analogy to this idea we write

$$
\left|\Psi_{B}(\alpha)\right\rangle=N_{B} \exp \left(-\alpha \hat{h}_{\mathrm{SSH}}\right)\left|\Psi_{\infty}\right\rangle,
$$

where $\alpha$ denotes the variational parameter and $\left|\Psi_{\infty}\right\rangle$ stands for a perfectly ordered charge density wave, and $N_{B}$ is a normalization constant. Our further calculations are rendered easier by the particularly simple form of $\left|\Psi_{\infty}\right\rangle$,

$$
\left|\Psi_{\infty}\right\rangle=\prod_{k \in \mathrm{RBZ}} \frac{1}{\sqrt{2}}\left(c_{k}^{\dagger}+c_{k+Q}^{\dagger}\right)|0\rangle
$$

where $Q=\pi$ is ordering wave vector (the lattice constant was assumed to be unity), the product runs through the reduced Brillouin zone (RBZ) and $|0\rangle$ is fermionic vacuum state. Fourier transforming the Hamiltonian results in

$$
\begin{aligned}
\hat{h}_{\mathrm{SSH}}(k) & =\left[\begin{array}{ll}
c_{k}^{\dagger} & c_{k+\pi}^{\dagger}
\end{array}\right]\left[\begin{array}{cc}
\epsilon(k) & \mathrm{i} \gamma(k) \\
-\mathrm{i} \gamma(k) & -\epsilon(k)
\end{array}\right]\left[\begin{array}{c}
c_{k} \\
c_{k+\pi}
\end{array}\right] \\
\hat{h}_{V} & =-\frac{V}{L} \sum_{k, k^{\prime}, q} \epsilon(q) c_{k+q}^{\dagger} c_{k} c_{k^{\prime}-q}^{\dagger} c_{k^{\prime}}
\end{aligned}
$$

where $\epsilon(k)=-t \cos k, \gamma(k)=-\delta \sin k$. With the help of the Pauli matrix representation of the SSH noninteracting Hamiltonian,

$$
\hat{h}_{\mathrm{SSH}}(k)=\epsilon(k) \sigma_{z}-\gamma(k) \sigma_{y}
$$

the SSH Hamiltonian based projector can be expanded as

$$
\exp \left(-\alpha \hat{h}_{\mathrm{SSH}}(k)\right)=\cosh (\alpha h(k)) \hat{\hat{I}}-\sinh (\alpha h(k)) \hat{\hat{h}}_{\mathrm{SSH}}(k)
$$

where $h(k)=\sqrt{t^{2} \cos ^{2} k+\delta^{2} \sin ^{2} k}, \hat{\hat{I}}$ is the 2-by-2 identity matrix, and

$$
\hat{\hat{h}}_{\mathrm{SSH}}(k)=\frac{\hat{h}_{\mathrm{SSH}}(k)}{h(k)},
$$

a unit vector in the space spanned by $\epsilon(k)$ and $\gamma(k)$. Now, applying the projector (6) on the CDW state (equation (3)) we obtain the normalized wave function,

$$
\left|\Psi_{B}(\alpha)\right\rangle=\prod_{k \in \mathrm{RBZ}} \frac{A(k) c_{k}^{\dagger}+A(k+Q) c_{k+Q}^{\dagger}}{\sqrt{2 \cosh [2 \alpha h(k)]}}|0\rangle,
$$

with

$$
A(k)=\cosh [\alpha h(k)]-\frac{\sinh [\alpha h(k)]}{h(k)} \epsilon(k)+\mathrm{i} \gamma(k) \frac{\sinh [\alpha h(k)]}{h(k)} .
$$

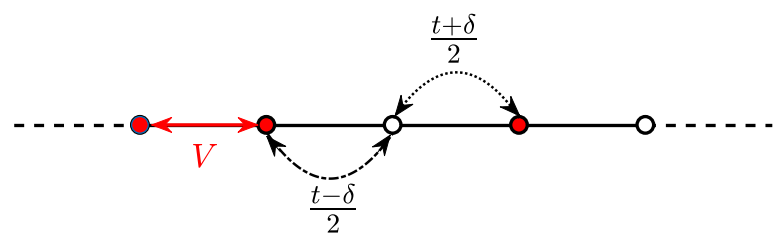

b)

Figure 1. Graphical representation of our model Hamiltonian (a) and the charge density wave (CDW) state (b). Filled (empty) circles indicate lattice sites occupied (not occupied) by particles. In (a) $V$ indicates the interaction, $t$ indicates the average hopping, $\delta$ denotes the amount by which the hopping alternates between even and odd bonds.

Having derived the action of the projector on the CDW state, we can now evaluate the variational estimate for the ground state energy by calculating the expectation value of equation (4) over our extended Baeriswyl wave function. We give the expectation values of the SSH Hamiltonian and of the interaction separately as

$$
\left\langle\hat{h}_{\mathrm{SSH}}\right\rangle=-\sum_{k} h(k) \tanh [2 \alpha h(k)]
$$

and

$$
\left\langle\hat{h}_{V}\right\rangle=\frac{V L}{4}-\frac{V}{L} \sum_{i=1}^{3}\left|T_{i}\right|^{2}
$$

where

$$
\begin{aligned}
& T_{1}=\sum_{k}\left[\frac{1}{2 \cosh [2 \alpha h(k)]}+\frac{\mathrm{i} \gamma(k)}{2 h(k)} \tanh [2 \alpha h(k)]\right] \\
& T_{2}=\sum_{k}\left[\frac{\epsilon(k)}{2}-\frac{\epsilon(k)^{2}}{2 h(k)} \tanh [2 \alpha h(k)]\right] \\
& T_{3}=\sum_{k}\left[\frac{\epsilon(k)}{2 \cosh [2 \alpha h(k)]}+\frac{\mathrm{i} \epsilon(k) \gamma(k)}{2 h(k)} \tanh [2 \alpha h(k)]\right] .
\end{aligned}
$$

For the $t-V$ model these expressions were derived in [50].

This newly derived wave function gives exact results in two extreme limits, $V \rightarrow 0$ and $V \rightarrow \infty$. In the former the total energy becomes equal to the energy of the SSH model, while in the latter the total energy tends to zero. In between these two opposite extreme limits a comparison of numerically calculated total energy with exact diagonalized calculation and optimal minimization parameter is given in the upper panel of figure 2 for a small sized system. The extended BWF gives results which compare well in all cases, although as $\delta$ increases, the agreement worsens.

\subsection{Parent Hamiltonian of the Baeriswyl wave function}

Visualizing topological phase transitions is greatly aided in the case of Hamiltonians which are two-state in $k$-space via plotting the curves traced out by sweeping through the Brillouin zone [51]. The standard way is to write the two-state Hamiltonian in the form $H(k)=\mathbf{h}(k) \cdot \sigma$, where $\mathbf{h}(k)$ is a 3D vector which traces out a closed curve in the space spanned by $h_{x}(k), h_{y}(k), h_{z}(k)$. 

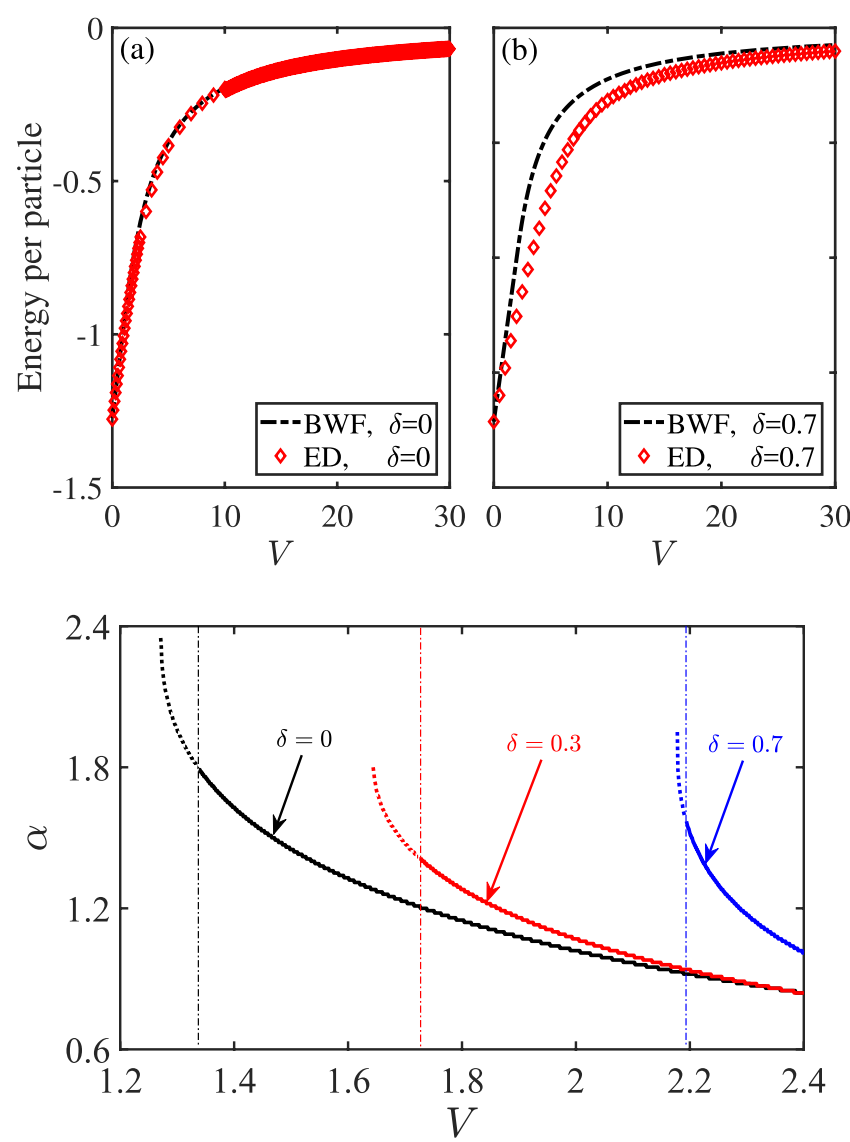

Figure 2. Upper panels: the variational ground state energy per particle for (a) $\delta=0$, and (b) $\delta=0.7$ based on the Baeriswyl wave function compared to exact diagonalization for 12 lattice sites. Lower panel: the variational parameter as a function of interaction strength for $(\delta=0, \pm 0.3)$ and $( \pm 0.7)$. Solid lines indicate the global minimum in the CDW type insulating phase. Dashed lines indicate metastable insulating phases on the metallic side of the phase diagram.

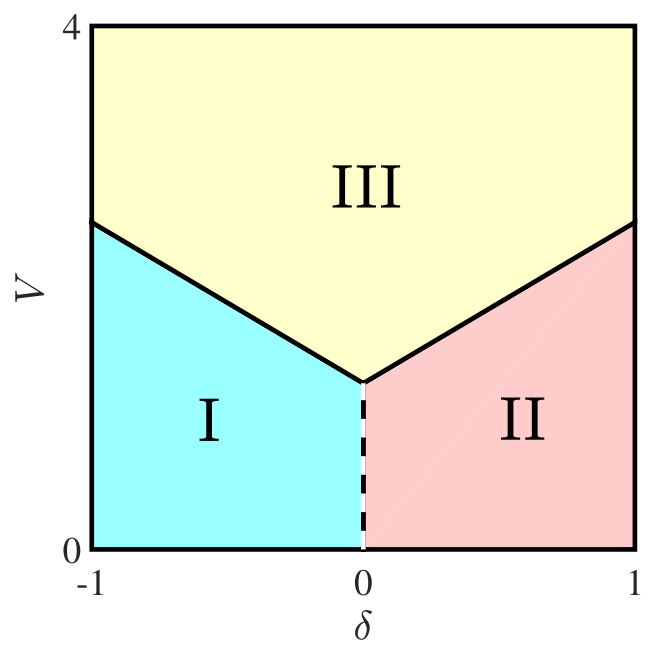

Figure 3. Phase diagram. I and II are SSH states (Hartree-Fock approximation). III is a CDW type phase, with finite skew when $\delta$ differs from zero. The dashed line indicates a Fermi sea (ideal conductor).

We can use the results of the previous subsection to construct an effective parent Hamiltonian for the BWF, which is also of the form $H(k)=\mathbf{h}(k) \cdot \sigma$. Our construction consists of two steps. First, inspecting equations (10)-(12), we are able to write the total energy (apart from the constant shift $V L / 4$ ) as a single sum over $k$, as $E=\sum_{k} \tilde{\epsilon}_{k}$. Since equation (12) includes, double $k$-sums, these contributions to $\tilde{\epsilon}(k)$ appear as single $k$ sums. Second, we consider the coefficients at $k$ and $k+Q$, and obtain the angles $\theta_{k}$ and $\phi_{k}$ according to the normalized equation

$$
\begin{aligned}
\tilde{\epsilon}_{k}\left[\begin{array}{cc}
\cos \left(\theta_{k}\right) & \sin \left(\theta_{k}\right) \mathrm{e}^{-\mathrm{i} \phi_{k}} \\
\sin \left(\theta_{k}\right) \mathrm{e}^{\mathrm{i} \phi_{k}} & -\cos \left(\theta_{k}\right)
\end{array}\right] & {\left[\begin{array}{c}
\frac{\sin \left(\theta_{k}\right)}{2} \\
-\frac{\cos \left(\theta_{k}\right)}{2} \mathrm{e}^{\mathrm{i} \phi_{k}}
\end{array}\right] } \\
& =-\tilde{\epsilon}_{k}\left[\begin{array}{c}
\frac{\sin \left(\theta_{k}\right)}{2} \\
-\frac{\cos \left(\theta_{k}\right)}{2} \mathrm{e}^{\mathrm{i} \phi_{k}}
\end{array}\right],
\end{aligned}
$$

where we equate

$$
\begin{aligned}
& \frac{A(k)}{\sqrt{2 \cosh [2 \alpha h(k)]}}=\frac{\sin \left(\theta_{k}\right)}{2} \\
& \frac{A(k+Q)}{\sqrt{2 \cosh [2 \alpha h(k)]}}=-\frac{\cos \left(\theta_{k}\right)}{2} \mathrm{e}^{\mathrm{i} \phi_{k}} .
\end{aligned}
$$

This procedure guarantees that summing over the RBZ gives the correct ground state energy, and that at each $k$-vector, the correct coefficients $A(k)$ and $A(k+Q)$ are obtained.

\subsection{Polarization and gauge invariant cumulants}

The gauge invariant cumulant series associated with the polarization was first studied by Souza et al [26]. In a general sense it can be derived [27] based on the discrete Berry phase (Bargmann invariant [52]). Here we give the basic expressions for the gauge invariant cumulants, for the reconstruction of the polarization we refer the reader to [28].

Consider a $1 \mathrm{D}$ system whose Hamiltonian is periodic in $L$. Taking periodic Bloch functions parametrized by the crystal momentum, $u(k)$, and defining $\gamma_{m}(k)=\left\langle u(k)\left|\partial_{k}^{m}\right| u(k)\right\rangle$, the first four cumulants take the form

$$
\begin{aligned}
& C_{1}=\mathrm{i} \frac{L}{2 \pi} \int_{-\frac{\pi}{L}}^{\frac{\pi}{L}} \mathrm{~d} K \gamma_{1} \\
& C_{2}=-\frac{L}{2 \pi} \int_{-\frac{\pi}{L}}^{\frac{\pi}{L}} \mathrm{~d} K\left[\gamma_{2}-\gamma_{1}^{2}\right] \\
& C_{3}=-\mathrm{i} \frac{L}{2 \pi} \int_{-\frac{\pi}{L}}^{\frac{\pi}{L}} \mathrm{~d} K\left[\gamma_{3}-3 \gamma_{2} \gamma_{1}+2 \gamma_{1}^{3}\right] \\
& C_{4}=\frac{L}{2 \pi} \int_{-\frac{\pi}{L}}^{\frac{\pi}{L}} \mathrm{~d} K\left[\gamma_{4}-3 \gamma_{2}^{2}-4 \gamma_{3} \gamma_{1}+12 \gamma_{1}^{2} \gamma_{2}-6 \gamma_{1}^{4}\right] .
\end{aligned}
$$

These quantities can be shown to be gauge invariant. If the Wannier functions associated with the Bloch functions are sufficiently localized, they correspond to the cumumlants of the probability distribution of the total position, and can be used in its reconstruction [28]. From the inversion of this cumulant series it is also possible to obtain gauge invariant moments. The cumulants can be inverted to obtain the gauge invariant moments, 

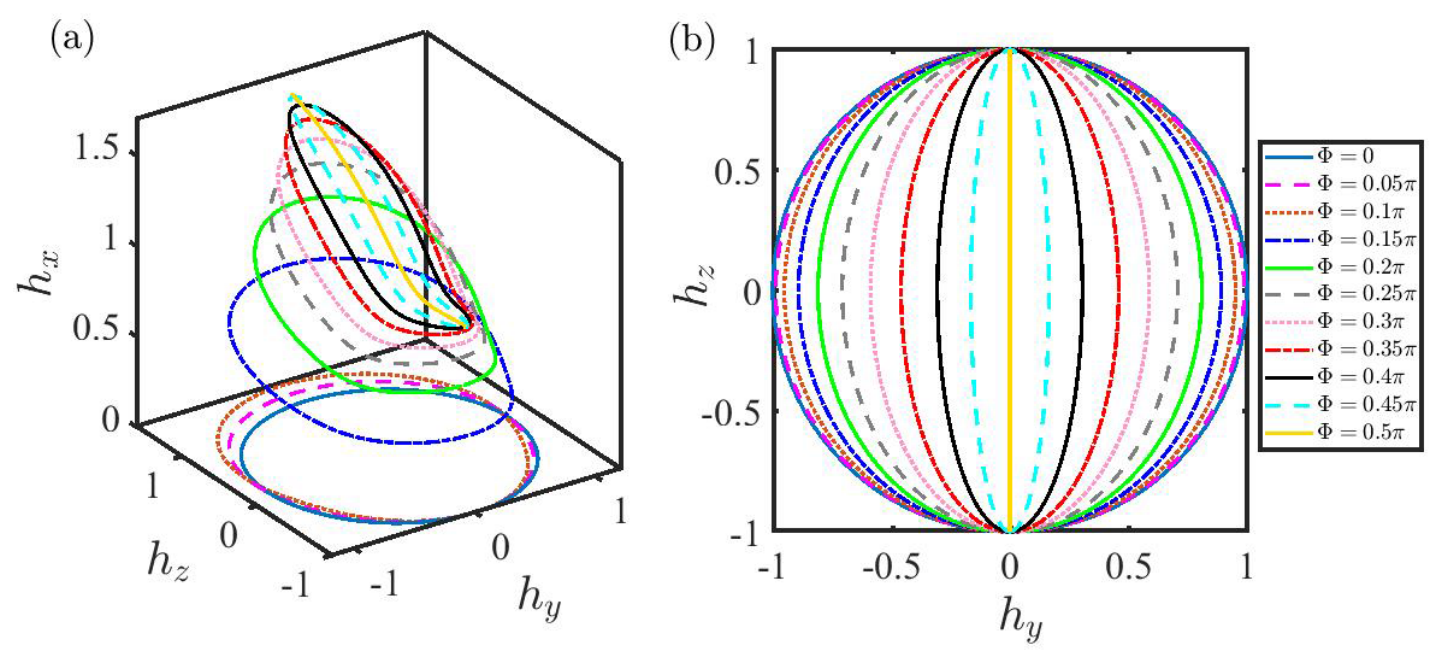

Figure 4. Curves traced out by the Hamiltonian in the Brillouin zone. (a) The correlated system, $\delta=\cos (\phi), V=1+4 \sin (\phi)$. In (b) $\delta=\cos (\phi), V=1$. For both panels, the values of the variable $\phi$ are indicated in the legend on the right.

$$
\begin{aligned}
& \mu_{C}^{(1)}=C_{1} \\
& \mu_{C}^{(2)}=C_{2}+C_{1}^{2} \\
& \mu_{C}^{(3)}=C_{3}+3 C_{2} C_{1}+C_{1}^{3} \\
& \mu_{C}^{(4)}=C_{4}+4 C_{3} C_{1}+3 C_{2}^{2}+6 C_{2} C_{1}^{2}+C_{1}^{4} .
\end{aligned}
$$

In our model the wave function is of the form given in equation (8). The cumulants can be obtained in a straightforward way, for example,

$$
\gamma_{m}(k)=A^{*}(k) \partial_{k}^{m} A(k)+A^{*}(k+Q) \partial_{k}^{m} A(k+Q) .
$$

Using $\gamma_{m}(k)$ the cumulants can be constructed according to equation (15), however the integrals and the normalization are now over the RBZ.

\section{Results and analysis}

The phase diagram of the model according to our calculations is shown in figure 3. For all values of $\delta$ the variational parameter at which the total energy is minimized is finite for large $V$ indicating a CDW type insulating phase. As $V$ decreases, at some $V_{t}$ (for a given $\delta$ ), the minimum at finite $V$ becomes a local minimum, with $\alpha=\infty$ becoming the global minimum, meaning that within our variational approximation the phase transition is first order (see figure 2). The $\alpha \rightarrow \infty$ limit corresponds to the SSH state for $V=0$, at finite $V$ it becomes the Hartree-Fock state, meaning the wave function is that of the SSH model, but the energy is not simply the SSH hopping energy, but also the expectation value of the interaction energy. A similar scenario was found in [48] for the Hubbard model. The small $V$ phase at $\delta=0$ is a Fermi sea, while for finite $\delta$ it is an SSH state. The metastable phase disappears at some finite $\delta$. Phase III is an insulating phase. When $\delta=0$ the skew is zero in this phase, when it is finite, a finite skew develops (see figure 5). Our phase diagram is qualitatively similar to the 2D KMH model, where the topologically non-trivial phases at small interaction become magnetically ordered insulating phases $[3,12]$.

We also calculated the curves traced out by the vector $\mathbf{d}$ which defines the parent Hamiltonian derived in section 2.3, shown in figure 4 . The left panel (a) shows the curve traced out by the Brillouin zone along specific points of a curve in the parameter space of the Hamiltonian which starts at $\delta=-1, V=1$ and ends at $\delta=1$ and $V=1$. The curve in between is an ellipse which does not cross the metallic phase line. The curves traced out our cyclic in 3D. At $\delta=0$ is approached the cyclic curves become 'thinner' and at $\delta=0$ itself the BZ is represented by a line rather than a cyclic curve. The right panel (b) shows what happens along the straight line $V=1$ but $\delta$ varying from -1 to 1 . This is exactly what happens in the SSH model, whose Hamiltonian is $d_{z}=-t \cos (k)$ and $d_{y}=-\delta \sin (k)$. As $\delta$ approaches to zero (gap closure) the curve becomes a line along the $y$-axis. The left panel gives some indication of the effect of correlation. Standard mean-field theory of the SSH model gives the RM model corresponding to an additional term in the $d_{x}$ directions with a renormalized on-site potential strength, and whose curves (not shown) would be similar to figure 4(b), but with a shift in the $d_{x}$ direction. One difference between mean-field theory and our BWF based treatment can be seen in the curves of figure 4(a).

In figure 5(a) we show the polarization $\left(C_{1}\right)$ on the $\delta-V$ plane. The polarization is take to be zero in the limit $V \rightarrow \infty$. As $V$ decreases the absolute value of the polarization increases, its sign depends on the sign of $\delta$. For finite $\delta$ the polarization saturates at a finite value of $\delta$. Particularly interesting is the behavior at $\delta=0$, where the polarization remains the same value, but we see that below $V<1.3365 \ldots$ it rises rapidly from $\delta<0$ to $\delta>0$, almost discontinuously. For $V>1.3365 \ldots$, the rise in $C_{1}$ across $\delta=0$ is smooth.

In figure 5(b) we show two paths we have chosen for our subsequent analysis. Paths $\mathcal{A}$ and $\mathcal{B}$ both connect the points $-1,1$ and 1,1 on the $\delta-V$ plane, but path $\mathcal{A}$ crosses the line segment $\delta=0,0<V<1.3365 \ldots$, while $\mathcal{B}$ does not, it passes 

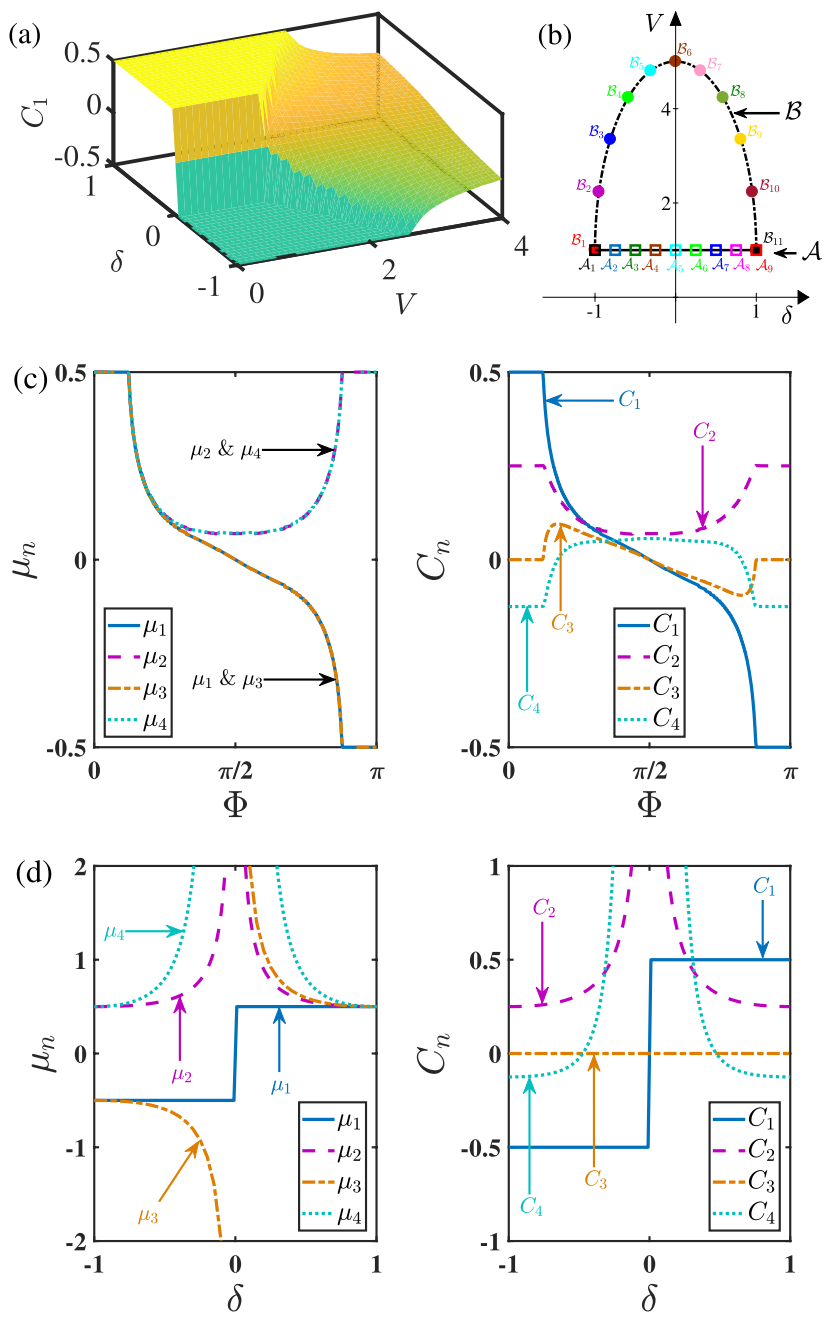

Figure 5. (a) First cumulant, $\left(C_{1}\right)$ or polarization, of the interacing SSH model as a function of $\delta$ and $V$. (b) Lines in the $\delta-V$ plane for which we calculate the cumulants and reconstruct the polarization. Two paths are shown between the points $-1,1$ and 1,1 : path $\mathcal{A}$ is a straight line, path $\mathcal{B}$ is a semi-ellipse. (c) Moments and cumulants along the semi-elliptic $(\mathcal{B})$ path. (d) Moments and cumulants along the straight line $(\mathcal{A})$ path.

$\delta=0$ above the point $V=1.3365 \ldots$. For the elliptical path the moments and cumulants are shown in part (c) of the figure. All cumulants and moments change continuously $C_{2}$ is minimum at $\delta=0$. In contrast to this, along the linear path $C_{1}$ changes discontinuously. $C_{2}$ increases sharply around $\delta=0$, indicating delocalization. All the cumulants change rapidly around $\delta=0$, the even cumulants are even functions, while the odd ones are odd.

In figure 6 we show reconstructed polarization distributions along a chosen set of points along the two different paths $\mathcal{A}$ and $\mathcal{B}$. Along the elliptical path $(\mathcal{B})$ the polarization distribution 'walks across' smoothly between the two positions related by symmetry. The distribution is a smooth function, the maximum changes continuously, and the shape of the distribution indicates a localized state (insulating). In contrast to this,
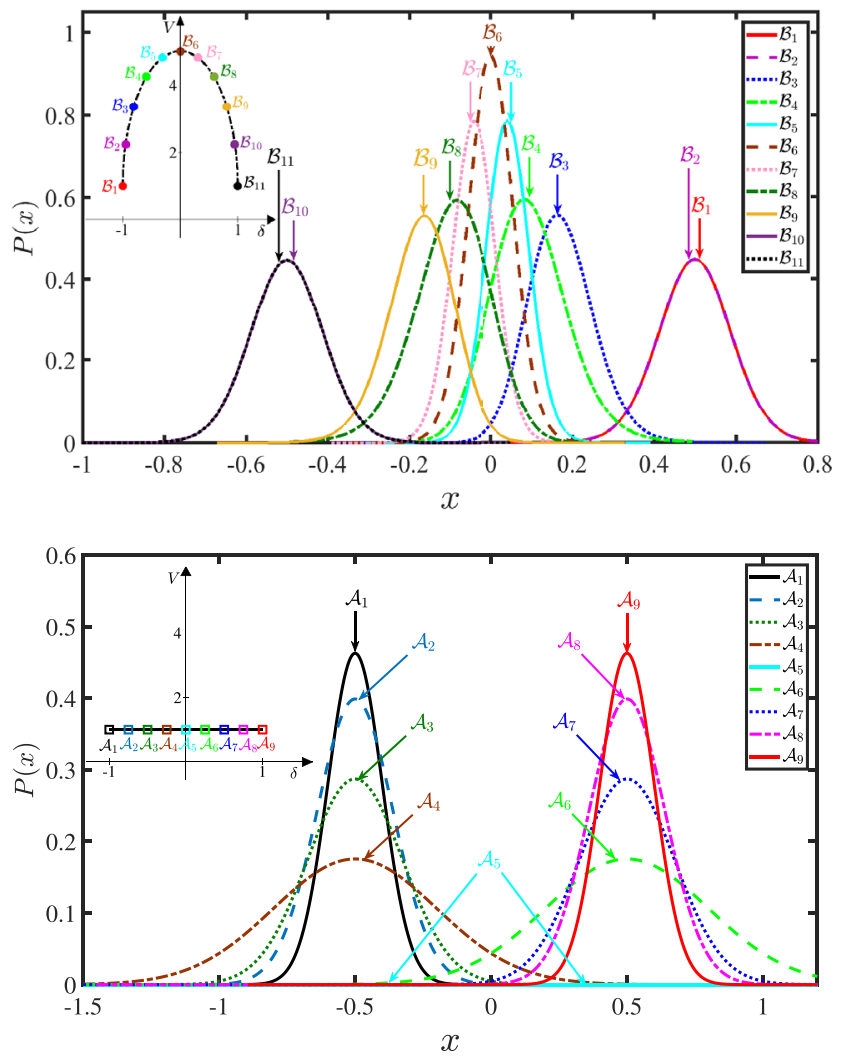

Figure 6. Reconstructed polarization distributions along the two paths shown indicated in figure 5. The upper panel (lower panel) shows reconstructed polarizations along the ellipse (straight line) between the points $-1,1$ to 1,1 on the $\delta-V$ plane.

when the polarization is reconstructed along the line which crosses the line $\delta=0,0<V<1.3365 \ldots$, the maximum of the distribution remains fixed in the interval $\delta>0$, while the width of the distribution is increasing (the distribution is becoming more delocalized). At $\delta=0$ the distribution is flat, the system is delocalized. We interpret this as a conducting state. For $\delta<0$ the distribution localizes around a different maximum. As the absolute value of $\delta$ increases the distribution becomes more localized.

We can relate the effect of the correlation to what happens around the topologically nontrivial point [19] of the SSH and RM models. We take as the definition of the RM model to be the SSH model defined above, plus an alternating onsite potential, which breaks the chiral symmetry, of the form $\Delta \sum_{j}(-1)^{j} c_{J}^{\dagger} c_{j}$, where $\Delta$ denotes the strength of the potential. In figure 7 we show reconstructed polarizations along a semi-circle on the $\delta-\Delta$ plane, and along a line along which the model is SSH, with a topological phase transition protected by symmetry. The gap closure (phase transition) point is at $\delta=\Delta=0$. The symmetry broken semi-circular path shows an evolution of the polarization distribution similar to the upper panel in figure 6 with the maximum shifting continuously, while along the linear path, the maximum shifts discontinuously between the $\delta<0$ and $\delta>0$ cases. 

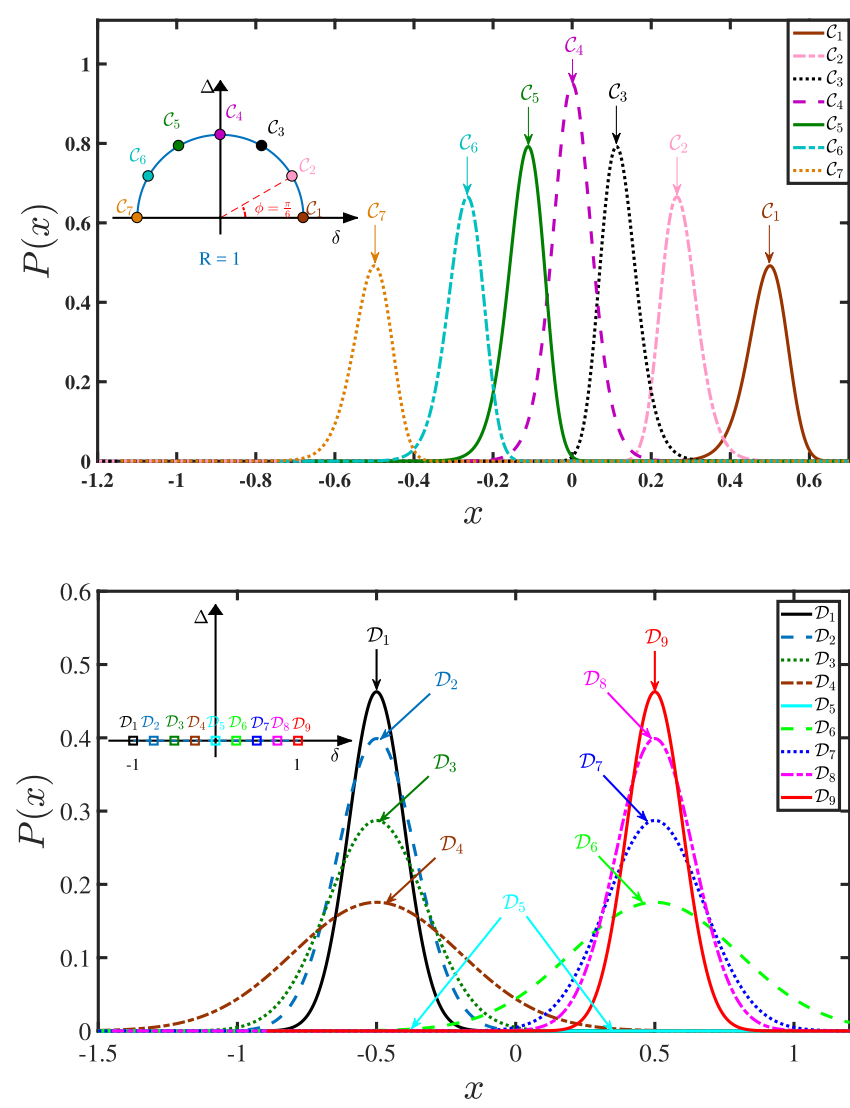

Figure 7. Reconstructed polarization for the RM model along the paths indicated in the inset of each plot. The paths are represented on the $\delta-\Delta$ plane.

\section{Conclusion}

We have developed a variational theory based on the Baeriswyl wave function for the SSH model with interaction, calculated the phase diagram of the model, and have studied the behavior of the polarization distribution as a function of the interaction. In our approximate scheme the transition is first order. Overall, the phase diagram is similar to the one found for another interacting topological model, the Kane-Mele-Hubbard model $[3,12]$.

It is worth mentioning that for the case of homogeneous hopping, an ideal conductor to insulator transition was found. This is surprising for a number of reasons. In general, variational wave functions for correlated systems are not thought to be flexible enough to produce phase transitions. For example, the Gutzwiller wave function is always metallic [53], and also for the Baeriswyl wave function, there exists a proof [47] that at finite value of the variational parameter it describes an insulating state. Our finding, however, is that a metal-insulator transition does occur, albeit, at a reduced value of the interaction $(V=1.3365 \ldots t)$ compared to the exact result $(V=2 t)$. Thus, for this model, the Baeriswyl wave function is qualitatively correct. Moreover, as far as the question of quantitivity is concerned, the ground state energy agrees well with exact diagonalization results.

For comparison, for the Hubbard model the exact result in one dimension is that there is no metal-insulator transition [54], and that it is insulating for finite interaction. The Baeriswyl wave function is thought to give this behavior as well, at least qualitatively, while the Gutzwiller wave function still only gives a metallic state. Thus, for both correlated models, the Hubbard model and the one treated in this work, the Baeriswyl wave function produces qualitatively the correct behavior.

Our study of the reconstructed polarization shows the effect of correlation on the phase diagram of the SSH model. It is instructive to compare the situation to the RM model, which is an SSH model with an on-site potential. This model exhibits a topologically non-trivial point (gap closure) at $\delta=0$ ( $\delta$ being the alternation in hoppings between odd and even bonds) and zero onsite potential. When interaction is added to the SSH model, the gap closure region is extended, it becomes a line, rather than just a point. Strictly speaking, gap closure is not accessible in our variational formalism, but the behaviour of the reconstructed polarization is identical. When a gap closure point, or line, is crossed, the polarization changes discontinuously, while if two states are connected by a path at which the gap does not close, the polarization evolves continuously. While our approach is variational, therefore approximate, we expect the qualitative picture to be robust, since the interaction gives rise to a symmetry broken state which is similar to the ground state of the RM model. In the RM model the charge density wave results from the alternating on-site potential. While, broadly speaking, our results suggests that turning on the interaction in the SSH model gives rise to similar effects as applying an alternating on-site interaction (RM), the difference is that the gap closure point of the non-interacting model becomes a line of points in the presence of interaction (also known from the exact solution of the model), while it is still a point in the parameter space in the RM case.

\section{Acknowledgments}

This research was supported by the National Research, Development and Innovation Fund of Hungary within the Quantum Technology National Excellence Program (Project Nr. 2017-1.2.1-NKP-2017-00001). BH thanks Balázs Dóra for helpful discussions.

\section{ORCID iDs}

M Yahyavi (i) https://orcid.org/0000-0003-0062-203X

B Hetényi (1) https://orcid.org/0000-0002-3680-1147

\section{References}

[1] Hasan M Z and Kane C L 2010 Rev. Mod. Phys. 823045

[2] Bernevig B A and Hughes T L 2013 Topological Insulators and Topological Superconductors (Princeton, NJ: Princeton University Press)

[3] Rachel S 2018 Rep. Prog. Phys. 81116501

[4] Gurarie V 2011 Phys. Rev. B 83085426

[5] Wang Z, Qi X-L and Zhang S-C 2010 Phys. Rev. Lett. 105256803 
[6] Budich J C, Thomale R, Li G and Laubach M 2012 Phys. Rev. B 86201407

[7] Budich J C, Trauzettel B and Sangiovanni G 2013 Phys. Rev. B 87235104

[8] Altland A and Zirnbauer M R 1997 Phys. Rev. B 551142

[9] Schnyder A P, Ryu S, Furusaki A and Ludwig A W W 2008 Phys. Rev. B 78195125

[10] Varney C N, Sun K, Rigol M and Galitski V 2010 Phys. Rev. B 82115125

[11] Jotzu G, Messer M, Desbuquois R, Lebrat M, Uehlinger T, Greif D and Esslinger T 2014 Nature 515237

[12] Rachel S and Le Hur K 2010 Phys. Rev. B 82075106

[13] Griset C and Xu C 2012 Phys. Rev. B 82045123

[14] Hamad I J, Gazza C J and Riera J A 2016 Phys. Rev. B 93205113

[15] Hohenadler M, Parisen Toldin F, Herbut I F and Assaad F 2014 Phys. Rev. B 90085146

[16] Laubach M, Reuther J, Thomale R and Rachel S 2014 Phys. Rev. B 90165136

[17] Berry M V 1984 Proc. R. Soc. A 39245

[18] Shapere A and Wilczek F 1989 Geometric Phases in Physics (Singapore: World Scientific)

[19] Xiao D, Chang M-C and Niu Q 2010 Rev. Mod. Phys. 821959

[20] Zak J 1989 Phys. Rev. Lett. 622747

[21] King-Smith R D and Vanderbilt D 1993 Phys. Rev. B 471651

[22] Resta R 1994 Rev. Mod. Phys. 66899

[23] Resta R 1998 Phys. Rev. Lett. 801800

[24] Thouless D J, Kohmoto M, Nightingale M P and den Nijs M 1982 Phys. Rev. Lett. 42405

[25] Fu L and Kane C L 2006 Phys. Rev. B 74195312

[26] Souza I, Wilkens T and Martin R M 2000 Phys. Rev. B 621666

[27] Hetényi B and Yahyavi M 2014 Europhys. Lett. 10540005

[28] Yahyavi M and Hetényi B 2017 Phys. Rev. A 95062104

[29] Resta R and Sorella S 1999 Phys. Rev. Lett. 82370

[30] Patankar S et al 2018 Phys. Rev. B accepted

[31] Kobayashi R, Nakagawa Y O, Fukusumi Y and Oshikawa M 2018 Phys. Rev. B 97165133
[32] Aligia A A and Ortiz G 1999 Phys. Rev. Lett. 822560

[33] Rice M J and Mele E J 1982 Phys. Rev. Lett. 491455

[34] Su W P, Schrieffer J R and Heeger A J 1979 Phys. Rev. Lett. 421698

[35] Yu W C, Li Y C, Sacramento P D and Lin H-Q 2016 Phys. Rev. B 94245123

[36] Krivnov V Ya and Ovchinnikov A A 1986 J. Exp. Theor. Phys. 39159

[37] Manmana S R, Essin A M, Noack R M and Gurarie V 2012 Phys. Rev. B 86205119

[38] Affleck I and Haldane F D M 1987 Phys. Rev. B 365291

[39] Schulz H J 1986 Phys. Rev. B 346372

[40] Yamanaka M, Hatsugai Y and Kohmoto M 1993 Phys. Rev. B 489555

[41] Nersesyan A A and Luther A 1994 Phys. Rev. B 50309

[42] Wang H T, Li B and Cho S Y 2013 Phys. Rev. B 8705442

[43] Haghshenas R, Langari A and Rezakhani A T 2014 J. Phys.: Condens. Matter 26456001

[44] Baeriswyl D 1986 Nonlinearity in Condensed Matter ed A R Bishop et al (Berlin: Springer)

[45] Baeriswyl D 2000 Found. Phys. 302033

[46] Valenzuela B, Fratini S and Baeriswyl D 2003 Phys. Rev. B 68045112

[47] Dzierzawa M, Baeriswyl D and Martelo L M 1997 Helv. Phys. Acta 70124

[48] Hetényi B 2010 Phys. Rev. B 82115104

[49] Hetényi B, Tanatar B and Martelo L M 2016 Phys. Rev. B 93174518

[50] Dóra B, Haque M, Pollmann F and Hetényi B 2016 Phys. Rev. B 93115124

[51] Asbóth J K, Oroszlány L and Pályi A 2016 A Short Course on Topological Insulators (Berlin: Springer)

[52] Bargmann V 1964 J. Math. Phys. 5862

[53] Millis A and Coppersmith S N 1991 Phys. Rev. B $4313770 \mathrm{R}$

[54] Lieb E and Wu F Y 1968 Phys. Rev. Lett. 21192 